\title{
Apocrine Hidradenocarcinoma of the Scalp: A Classification Conundrum
}

\author{
Marc Cohen · David S. Cassarino · Hubert B. Shih • \\ Elliot Abemayor · Maie St. John
}

Received: 22 October 2008/Accepted: 20 November 2008/Published online: 5 December 2008

(C) The Author(s) 2008. This article is published with open access at Springerlink.com

\begin{abstract}
Introduction The classification of malignant sweat gland lesions is complex. Traditionally, cutaneous sweat gland tumors have been classified by either eccrine or apocrine features. Methods A case report of a 33-yearold Hispanic man with a left scalp mass diagnosed as a malignancy of adnexal origin preoperatively is discussed. After presentation at our multidisciplinary tumor board, excision with ipsilateral neck dissection was undertaken. Results Final pathology revealed an apocrine hidradenocarcinoma. The classification and behavior of this entity are discussed in this report. Conclusion Apocrine hidradenocarcinoma can be viewed as an aggressive malignant lesion of cutaneous sweat glands on a spectrum that involves both eccrine and apoeccrine lesions.
\end{abstract}

Keywords Apocrine $\cdot$ Eccrine $\cdot$ Hidradenocarcinoma . Sweat gland $\cdot$ Scalp $\cdot$ Head $\cdot$ Neck

\section{Introduction}

The skin is the largest organ of the body and is composed of two principal layers, the epidermis and dermis. Arising

M. Cohen $(\bowtie) \cdot$ E. Abemayor · M. St. John

Division of Head and Neck Surgery, UCLA School of Medicine, 10838 Le Conte Ave, Rm 62-132 CHS, Los Angeles, CA 90095,

USA

e-mail: macohen@mednet.ucla.edu

D. S. Cassarino

Department of Pathology and Laboratory Medicine, UCLA

School of Medicine, Los Angeles, CA, USA

H. B. Shih

UCLA School of Medicine, Los Angeles, CA, USA out of the ectoderm and eventually existing within the dermis are specialized structures referred to as skin appendages. Skin appendages are subdivided into three categories: the pilosebaceous unit, the eccrine cutaneous sweat gland, and the apocrine cutaneous sweat gland. Eccrine sweat glands are found throughout the body but especially on the palms and soles. Apocrine sweat glands tend to be limited to the face, axilla, and anogenital areas [1].

Skin adnexal tumors can arise from any of these skin appendages. For example, pilosebaceous units are thought to give rise to trichilemmal carcinoma and sebaceous carcinoma. Some examples of tumors of supposed eccrine origin include porocarcinoma and hidradenocarcinoma, though apocrine differentiation of the former has been seen as well. Apocrine carcinoma and syringocystadenocarcinoma are thought to have apocrine origins. Hence, there usually exists a pilosebaceous, eccrine, or apocrine predominant morphology which allows classification of skin adnexal tumors into one of these three groups of origin $[1,2]$.

As previously mentioned, hidradenocarcinoma is traditionally regarded as a malignancy of eccrine cutaneous sweat glands. In this report, we present a case of a patient with a hidradenocarcinoma with apocrine features. The dilemma of classifying this lesion and its behavior will be discussed.

\section{Case Report}

A 33-year-old man presented with a 4 month history of an enlarging left scalp mass. The patient denied any pain or parasthesias associated with the mass or any history of trauma to the area. He did note that the mass would 
occasionally bleed. He had no history of significant sun exposure, alcohol or tobacco use.

Physical examination revealed a $5 \times 5 \times 4 \mathrm{~cm}$ painless mass at the left posterior scalp (Fig. 1). The mass appeared to be extremely vascular with normal overlying skin. The mass did not feel to be fixated to the underlying calvarium. In addition, multiple enlarged left level $\mathrm{V}$ lymph nodes were palpated.

The mass was evaluated by both computed tomography (CT) and magnetic resonance (MR). On CT, the mass was clearly identified and noted not to have an calvarial involvement. By MR imaging, the mass was relatively isointense on $\mathrm{T} 1$ weighted images, slightly bright on $\mathrm{T} 2$ weighted images with flow voids (Fig. 2). On both MR and $\mathrm{CT}$, the presence of significant left-sided cervical adenopathy was evident. A PET scan was also obtained which showed abnormal uptake at the left posterior scalp and mid-left lower neck. No distant lesions were identified.

Incisional biopsies were performed and a diagnosis of a malignant adnexal neoplasm was given. The patient was presented at our multidisciplinary tumor board and the recommendation was to perform a wide local excision of the primary mass and a modified radical neck dissection of the ipsilateral neck. The decision to give postoperative adjuvant treatment would be made after final pathology.

The patient underwent wide local excision of the mass and a left neck dissection of levels I-V. Surgical margins were achieved. On microscopic exam, the mass was consistent with an infiltrative carcinoma with clear cell morphology and focal squamous differentiation, with focal sebaceous differentiation and connection to a hair follicle, numerous duct-like structures, and myxoid stroma (Figs. 3 and 4). Immunohistochemical staining was grossly positive for HMW-cytokeratin (Fig. 5) and focally positive for CEA. CAM 5.2, CK7, CK20, S-100, TTF-1, and CDX2

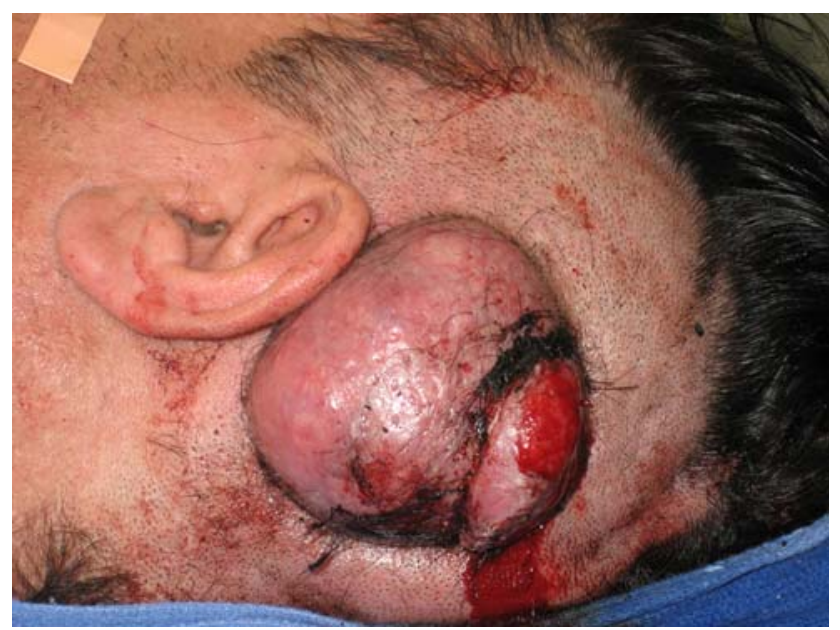

Fig. 1 After the patient's scalp has been shaved, the $5 \times 5 \times 4 \mathrm{~cm}$ mass at the left parietal scalp as seen upon initial presentation were all negative. A final diagnosis of apocrine hidradenocarcinoma was made.

\section{Discussion}

Although adnexal malignancies comprise $<1 \%$ of all head and neck cancers, it is nevertheless important for the head and neck surgeon to understand the classification and behavior of these tumors. As mentioned, adnexal tumors have been classified based on their predominant morphology. Traditionally, these tumors were categorized as having a pilosebaceous, eccrine, or apocrine origin.

However, some investigators have pointed to the existence of tumors of mixed morphology, such as tumors exhibiting both apocrine and eccrine differentiation. This has prompted the suggestion that certain skin adnexal tumors may arise out of a totipotent stem cell instead [1]. More relevantly, such observations have prompted some to question the usefulness and accuracy of traditional designations of cutaneous sweat gland tumors as either of eccrine or apocrine in origin [2-4]. The traditional association of hidradenocarcinoma with eccrine morphology compared to our current observation of apocrine hidradenocarcinoma certainly supports this line of thought.

Several other examples help explain the motivation behind questioning traditional eccrine and apocrine designations. First is the aforementioned example of the existence of mixed eccrine/apocrine lesions such as syringocystadenoma papilliferum and poroma [2]. Second, many tumors which have been previously classified as apocrine have been found in areas where apocrine glands typically do not exist [3]. Third, closer examination of many lesions traditionally classified as eccrine have revealed the existence of apocrine and apoeccrine counterparts as well [2]. Given that many past examples of eccrine or apocrine designation of cutaneous sweat gland tumors were based on poor or incomplete evidence, Ko and colleagues undertook a study carefully examining the histological and immunohistochemical characteristics of a series of six hidradenocarcinomas. Their conclusion was that all the samples demonstrated histological features consistent with both eccrine and apocrine differentiation [3]. Fourth is the observation that that there exists apoeccrine glands in the axillary region and also glands called cutaneous mammary-like glands in the anogenital region that demonstrate features of all three skin appendages. Because of all these reasons, combined apoeccrine designation has been proposed for many cutaneous sweat gland tumors including the hidradenocarcinoma [2, 3].

In addition, many tumors show features suggestive of folliculosebaceous apocrine unit (FSAU) derivation, with both follicular and/or sebaceous areas as well as ductal 
Fig. 2 Axial T1 (a) and coronal T2 (b) MR images of the left parietal scalp mass
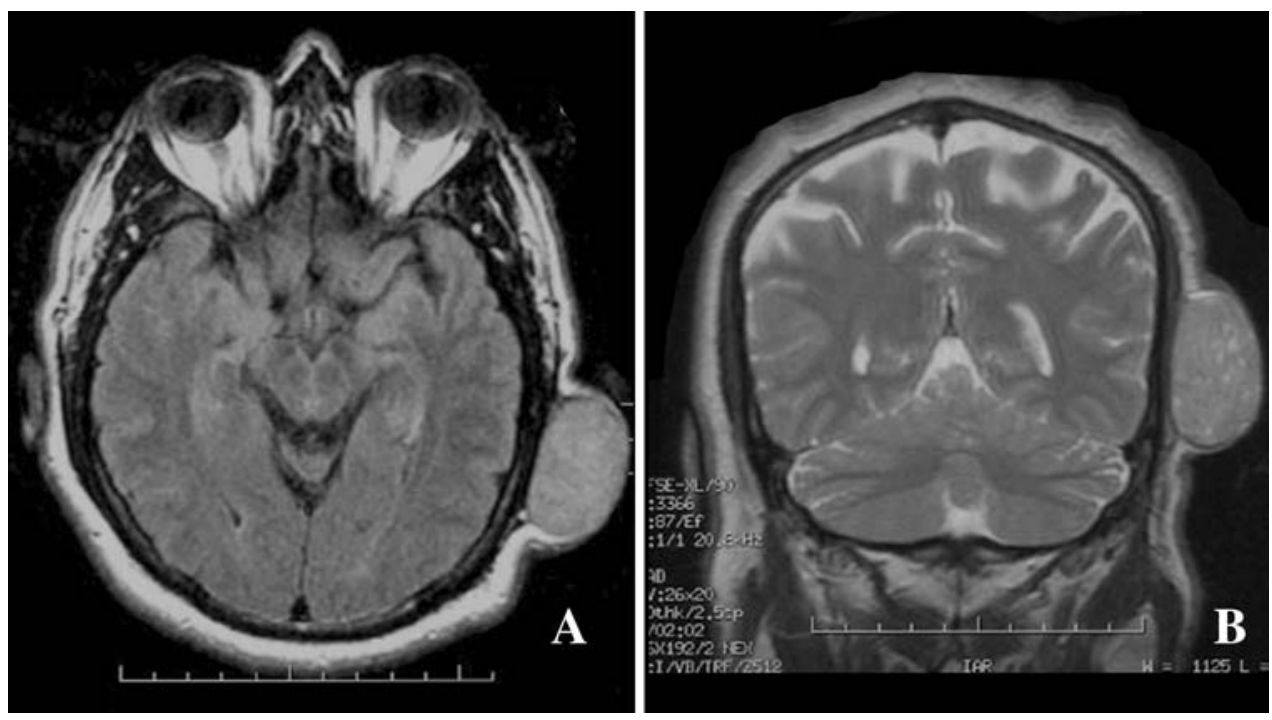

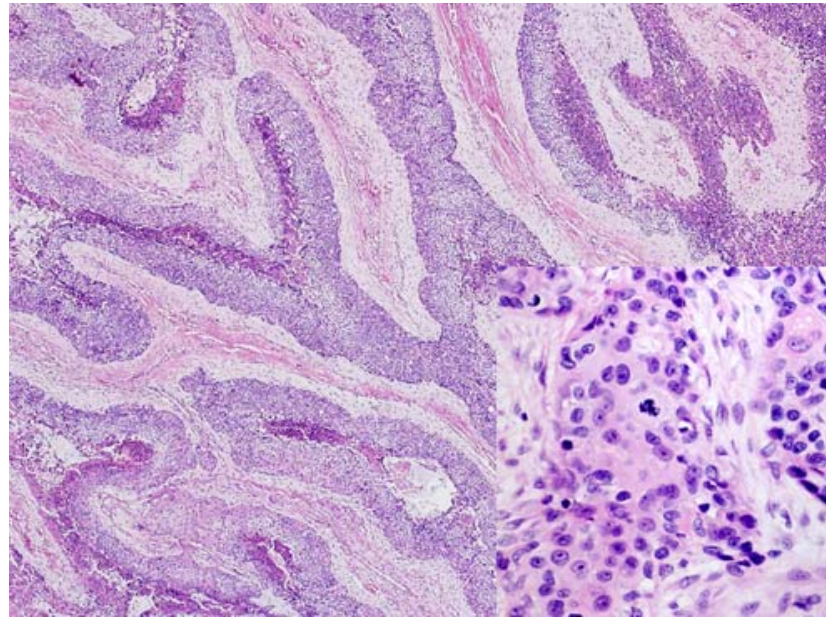

Fig. 3 Histologic examination showed an infiltrative-appearing, dermal neoplasm composed of anastomosing cords of eosinophilic to clear-staining tumor cells with areas of necrosis. High power examination showed cytologic atypia with enlarged nucleoli and easily identifiable mitoses (inset)

areas, which are most likely apocrine [4]. Hidradenocarcinoma and hidradenoma, its benign counterpart, may be FSAU derived in some cases.

Nonetheless, many pathologists have continued to classify cutaneous sweat gland tumors by their predominant morphology and the designations of eccrine, apocrine, and FSA remain consistently used. In general, some characteristics of eccrine glands include the following: excretory coil cells are positive for low molecular weight keratin, epithelial membrane antigen, and carcinoembryonic antigen; the myoepithelial layer may demonstrate smooth muscle actin, p63, and calponin; cells in the intraepidermal portion are positive for high molecular weight keratin and cytokeratin 14. In addition, some cells are also positive for bcl-2 and estrogen and progesterone receptors. The clinical implication of this is that hormonal therapy may potentially be used as part of the treatment of such eccrine tumors [2].

In contrast, pure apocrine lesions are usually negative for bcl-2 and estrogen and progesterone receptors, though its glandular epithelium is often positive for androgen receptors. Apocrine lesions tend to be found where apocrine glands are concentrated, such as the axilla and anogenital areas, but they can also be found in other areas such as the umbilicus, eyelid, areola, and external auditory meatus. Such lesions usually demonstrate abundant eosinophilic cytoplasm and have PAS-positive diastase-resistant granules and iron pigment. Eccentric, basally located nuclei and decapitation secretion are also commonly seen. Its luminal cells are positive for epithelial membrane antigen and carcinoembryonic antigen, while its secretory cells are positive for low molecular weight keratin [2].

As mentioned, case reports of hidradenocarcinoma have predominantly detailed lesions of supposed eccrine morphology. This is perhaps partly due to the possibility that many supposedly eccrine lesions are truly apocrine, apoeccrine, or FSAU derived lesions. In addition, hidradenocarcinoma, to begin with, is extremely rare. However, apocrine differentiation does occur. The histological and immunohistochemical profile of the hidradenocarcinoma can span a wide spectrum and may include various markers typically ascribed to eccrine and apocrine glands as described above. Reflecting this wide spectrum is the variety of names that have been used to describe the tumor: malignant nodular/clear cell hidradenoma, malignant acrospiroma, malignant clear cell acrospiroma, and clear cell eccrine carcinoma [2].

Hidradenocarcinoma was first described in 1954 by Keasby and Hadley as a clear cell eccrine carcinoma. Reflecting its rarity, hidradenocarcinomas have been 
Fig. 4 a The tumor showed evidence of ductal differentiation with numerous patent ductal lumina (arrows). b In addition, areas of follicular differentiation were also apparent, as the tumor showed attachment to several follicularcystic structures
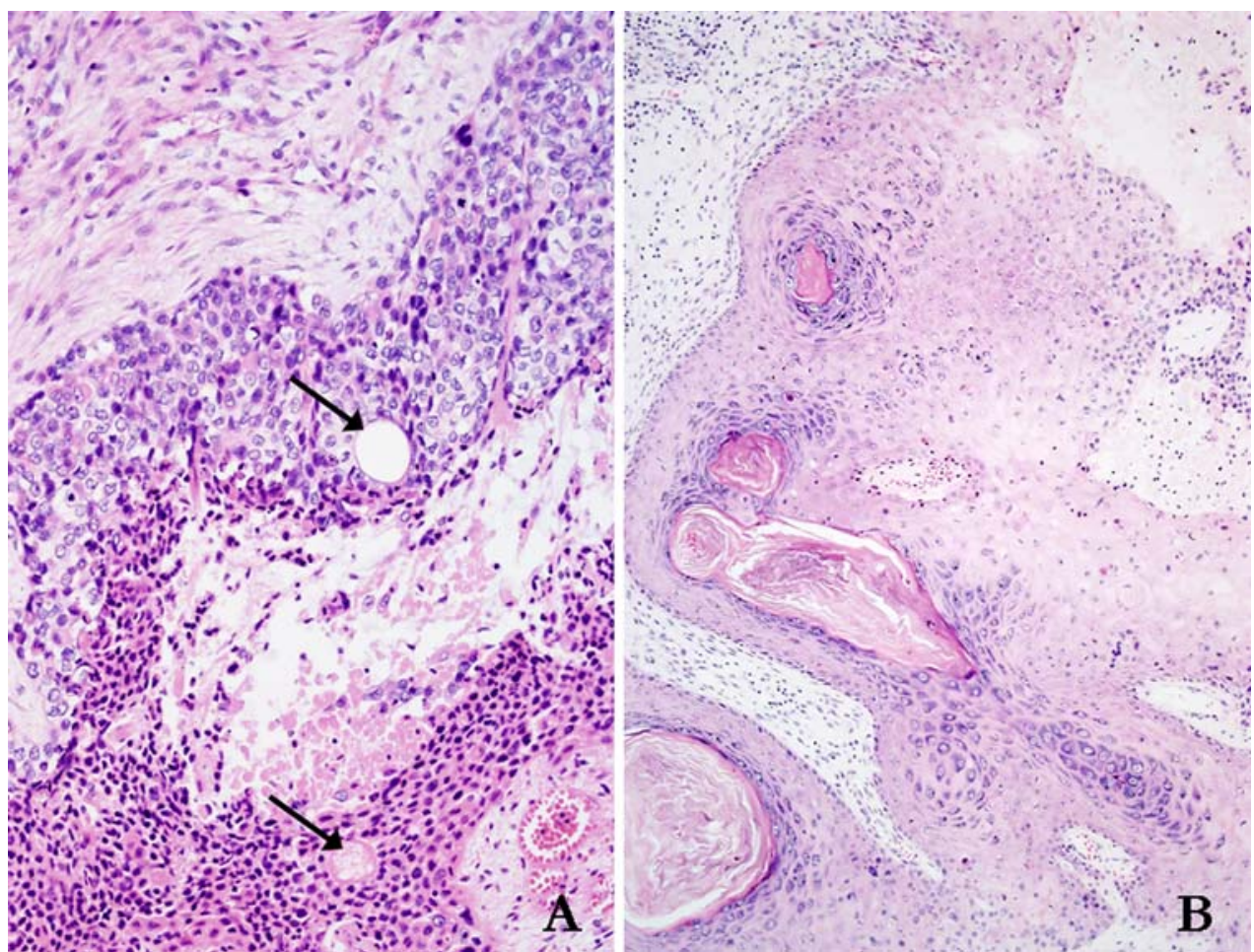

described in the literature fewer than 60 times since then [5]. It usually presents on the face or limbs as an ulcerated reddish nodule in elderly patients [6]. The tumor is most distinctly characterized by its frequent clear cell morphology. It is a multinodular solid malignant neoplasm that demonstrates ductal structures and intracytoplasmic tubular vacuoles along with areas of invasion and may show tumor necrosis. It most often arise de novo but may also arise out of its benign counterpart (hidradenoma). Both cytonuclear atypia and increased mitotic activity, in addition to infiltrative borders, vascular and neural invasion, and lymph node metastases, may help distinguish it from
Fig. 5 The tumor cells were diffusely positive for high molecular weight cytokeratin (a), and focally positive in the ductal areas for CEA (b)

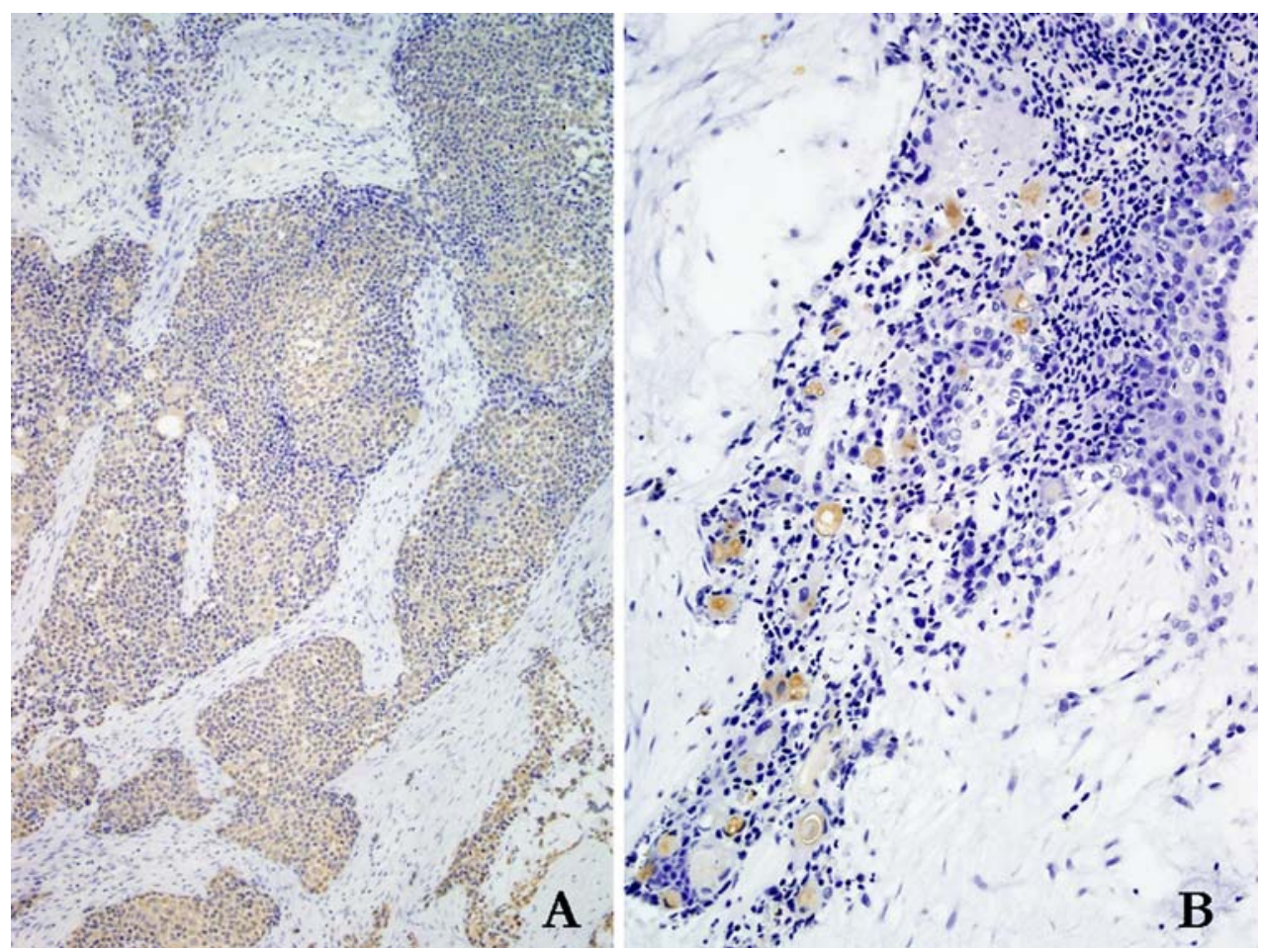


hidradenoma. Macroscopically, compared to its benign counterpart, hidradenocarcinoma appears to be larger, less well demarcated, and more asymmetrical $[2,5,7]$.

The differential diagnosis for hidradenocarcinoma primarily includes tumors exhibiting malignant characteristics with clear cell cytology. These include clear cell eccrine carcinoma and porocarcinoma, sebaceous carcinoma, tricholemmal carcinoma, clear cell squamous cell carcinoma, balloon cell melanoma, and clear cell basal cell carcinoma [5, 8]. Clear cell carcinomas metastatic to the skin such as metastatic renal cell clear cell carcinoma should also be considered [5].

Another adnexal malignancy with apocrine features that must also be considered is apocrine carcinoma. Primary cutaneous apocrine carcinoma is a rare tumor with infrequent reports in the literature. This lesion, like hidradenocarcinoma, has a very high rate of regional lymph node metastases and recurrence. Despite these aggressive features, apocrine carcinoma is thought to have a good prognosis since it is infrequently fatal [9].

In contrast, the prognosis for hidradenocarcinoma is considered to be poor. Even with aggressive surgical management, the rate of local recurrence has been seen to be anywhere from 10 to $50 \%$ [10, 11]. Regional lymph nodes are a common route of metastases; indeed, in more than $60 \%$ of patients, metastases have been reported within the first 2 years. Hematogenous metastases to periesophageal, peribronchial, periaortic, retroperitoneal lymph nodes, bones, vertebrae, ribs, pelvis, lung space, viscera, and pleura have been reported as well. In general, the prognosis for 5 year disease-free survival is $<30 \%$ [7].

In addition to aggressive surgical excision (which is generally considered to be the standard treatment), some have recommended removal of regional lymph nodes due to the high rates of metastases [11]. However, partly due to the rare nature of the tumor, the role of lymph node dissection, sentinel lymph node biopsy, and lymphadenectomy on survival remains to be determined, as does the role of adjuvant chemotherapy. Reports on the effectiveness of radiotherapy have been conflicting, with some reports showing radiotherapy to be an effective treatment and other reports demonstrating radio-resistance $[5,7]$.

The use of Mohs micrographic surgery has also been reported. Wildemore et al reported three patients with hidradenocarcinoma of the lower back, groin, and scalp treated with Mohs surgery to be free of any evidence of metastatic disease at 44,41 , and 30 months respectively [10].
In summary, apocrine hidradenocarcinoma can be viewed as an aggressive malignant lesion of cutaneous sweat glands on a spectrum that involves both eccrine and apoeccrine or FSAU derived lesions. These lesions, in most cases, may be derived from a pluripotent precursor cell with the capacity for multiple lines of adnexal differentiation, which could account for the morphologic variability of these tumors. However, the fact that hidradenocarcinomas, regardless of their degree of apocrine or eccrine differentiation, show a similar aggressive behavior, should dictate that these lesions be treated in a similar fashion. Although the data is limited, the treatment of these lesions should include aggressive surgical excision with regional lymphadenectomy and adjuvant therapy.

Open Access This article is distributed under the terms of the Creative Commons Attribution Noncommercial License which permits any noncommercial use, distribution, and reproduction in any medium, provided the original author(s) and source are credited.

\section{References}

1. Alsaad KO, Obaidat NA, Ghazarian D. Skin adnexal neoplasmspart 1: an approach to tumours of the pilosebaceous unit. J Clin Pathol. 2007;60:129-44.

2. Obaidat NA, Alsaad KO, Ghazarian D. Skin adnexal neoplasmspart 2: an approach to tumours of cutaneous sweat glands. J Clin Pathol. 2007;60:145-59.

3. Ko CJ, Cochran AJ, Eng W, Binder SW. Hidradenocarcinoma: a histological and immunohistochemical study. J Cutan Pathol. 2006;33:726-30.

4. Mattoch I, Cassarino D. Cutaneous Adnexal Neoplasms With Eccrine or Apocrine Differentiation: Diagnostic Distinction, and Does It Really Matter? Pathology Case Reviews. 2007;12:55-60.

5. Verret DJ, Kabbani W, DeFatta RJ. Nodular hidradenocarcinoma over the parotid gland: a pathologic presentation. Head Neck. 2007;29:193-7.

6. Bogner PN, Fullen DR, Lowe L, et al. Lymphatic mapping and sentinel lymph node biopsy in the detection of early metastasis from sweat gland carcinoma. Cancer. 2003;97:2285-9.

7. Ohta M, Hiramoto M, Fujii M, Togo T. Nodular hidradenocarcinoma on the scalp of a young woman: case report and review of literature. Dermatol Surg. 2004;30:1265-8.

8. Crowson AN, Magro CM, Mihm MC. Malignant adnexal neoplasms. Mod Pathol. 2006;19(Suppl 2):S93-126.

9. Robson A, Lazar AJ, Ben Nagi J, et al. Primary cutaneous apocrine carcinoma: a clinico-pathologic analysis of 24 cases. Am J Surg Pathol. 2008;32:682-90.

10. Wildemore JK, Lee JB, Humphreys TR. Mohs surgery for malignant eccrine neoplasms. Dermatol Surg. 2004;30:1574-9.

11. Khalil HM, Yusuf H, Kaddour HS. Malignant eccrine hidradenoma of neck causing acute heart failure. Auris Nasus Larynx. 2003;30:307-10. 